\title{
Expression levels of JNK associated with polymorphic lactotransferrin haplotypes in human nasopharyngeal carcinoma
}

\author{
GENGQIU LUO ${ }^{1 *}$, YANHONG ZHOU $^{2 *}$, WEI $\mathrm{YI}^{2}$ and $\mathrm{HONG} \mathrm{YI}^{3}$ \\ ${ }^{1}$ Department of Pathology, Xiangya Hospital, Central South University, Changsha, Hunan 410008; \\ ${ }^{2}$ Molecular Genetics Laboratory, Cancer Research Institute, Central South University, Changsha, Hunan 410078; \\ ${ }^{3}$ Research Center of Carcinogenesis and Targeted Therapy, Xiangya Hospital, Central South University, \\ Changsha, Hunan 410008, P.R. China
}

Received January 5, 2015; Accepted February 12, 2016

DOI: $10.3892 / 01.2016 .4723$

\begin{abstract}
Lactotransferrin (LTF), a member of the transferrin family, serves a role in the innate immune response and is involved in anti-inflammatory, anti-microbial and anti-tumor activity. Alterations in the LTF gene are associated with an increased incidence of cancer. The $L T F$ gene is polymorphic, and several common alleles may be observed in the general population. Our previous study identified a lower rate of occurrence of the 'A-G-G-T' haplotype (constructed with rs1126477, rs1126478, rs2073495 and rs9110) in nasopharyngeal carcinoma (NPC) patients compared with controls. In the present study, in order to elucidate a possible mechanism of LTF-mediated anti-tumor activity in NPC, the protein profiles of NPC and non-tumorous nasopharyngeal epithelium tissues with/without the 'A-G-G-T' haplotype were constructed using LTQ Orbitrap technology. The results revealed that c-Jun N-terminal kinase 2 (JNK2) was highly expressed in NPC tissues and non-tumor nasopharyngeal epithelium tissues without the 'A-G-G-T' haplotype. These results were confirmed by western blot analysis. Furthermore, microRNA (miRNA) microarray analysis was conducted to investigate the differential miRNA profiles of NPC and non-tumor nasopharyngeal epithelium tissues with/without the 'A-G-G-T' haplotype. It was observed that hsa-miR-1256 and hsa-miR-659, which are potentially targeted to the $J N K 2$ gene, were downregulated in
\end{abstract}

Correspondence to: Professor Hong Yi, Research Center of Carcinogenesis and Targeted Therapy, Xiangya Hospital, Central South University, 87 Xiangya Road, Changsha, Hunan 410008, P.R. China

E-mail: yihongxy2014@163.com

*Contributed equally

Abbreviations: NPC, nasopharyngeal carcinoma; LTF, lactotransferrin; JNK2, c-Jun N-terminal kinase 2; GADPH, glyceraldehyde-3-phosphate dehydrogenase

Key words: JNK2, nasopharyngeal carcinoma, lactotransferrin, expression
NPC tissues without the 'A-G-G-T' haplotype. Hsa-miR-298, another miRNA potentially targeted to the JNK2 gene, was downregulated in non-tumor nasopharyngeal epithelium tissues without the 'A-G-G-T' haplotype. In summary, these results suggested that the expression levels of JNK2 may be associated with polymorphic LTF haplotypes in human NPC.

\section{Introduction}

Under normal conditions, lactotransferrin (LTF) may be detected in human blood, mucosal secretions, gastrointestinal fluids, urine and, primarily, in milk and colostrum (1). LTF is a member of the transferrin family that has roles in the innate immune response and is involved in anti-inflammatory, anti-tumor and anti-microbial activity (2-9). The LTF gene is polymorphic, and several common alleles occur in the general population (3,7-9). Alterations in the LTF gene in cells are associated with an increased incidence of cancer (10). Our previous study found that NPC patients had lower rate of the 'A-G-G-T' haplotype (composed of rs1126477, rs1126478, rs2073495 and rs9110) compared with cancer-free controls. The population with the 'A-G-G-T' haplotype had a 0.322 -fold risk of having nasopharyngeal carcinoma (NPC). Thus, this haplotype is a protective factor (3).

NPC has a distinctive ethnic and geographical distribution, exhibiting a prevalence among the southern Chinese population with an incidence rate 25 -fold higher than the majority of other countries (11). The predominant etiological factors that contribute to the development of NPC comprise infection with Epstein-Barr virus, exposure to chemical carcinogens or radiation, functional or structural mutation of oncogenes and tumor suppressor genes, and chromosomal aberrations (2-6). Some evidence regarding the molecular bases of NPC has been reported; however, it remains to be fully clarified. Thus, it is urgent to elucidate the possible role and mechanism of LTF in NPC.

In multiple myeloma, Stella et al (12) observed that heterozygous carriers of the glutathione S-transferase P1 gene had reduced expression compared to those with the homozygous wild type genotype. Velliyagounder et al (13) found that a Lys/Arg polymorphism (rs1126478) at position 29 in the N-terminal region of human LTF produced functional differences, and 
that this difference may contribute to the pathogenesis of localized juvenile periodontitis. Similarly, control individuals who were G carriers at rs1126478 had significantly lower fasting triglyceride concentrations and significantly higher high-density lipoprotein cholesterol concentrations than AA homozygotes (14). This evidence suggests that polymorphism of genes may be associated with the expression of other genes.

In the current study, to identify the molecules associated with polymorphisms of $L T F$, the protein and microRNA (miRNA) profiles of NPC and non-tumor nasopharyngeal epithelium tissues with/without the 'A-G-G-T' haplotype were constructed.

\section{Materials and methods}

Patient samples. A total of 40 participants were recruited between April and December 2013 at the Cancer Hospital of Hunan Province in Changsha (China). Written informed consent was obtained from individual patients, and experimental protocols were approved by the Institutional Review Board of the Cancer Hospital of Hunan Province. Patients had histologically-confirmed epithelial NPC $(n=20)$ or chronic inflammation of the nasopharyngeal mucosa $(n=20)$, and were living in Hunan Province within one year of diagnosis. All subjects enrolled in the study were Chinese. There was no significant difference in distribution of gender and age between NPC patients and those with non-tumorous nasopharyngeal epithelium (Table I). Biopsy tissue samples of NPC and chronic inflammation of nasopharyngeal mucosa were collected, in addition to peripheral blood samples, and each biopsy sample was divided into two sections: One was submitted for routine histological diagnosis, and the remaining section was flash-frozen and stored at $-80^{\circ} \mathrm{C}$ in liquid nitrogen.

Blood collection and genotyping. An extra vial of blood was drawn from the participants during their scheduled medical visit. DNA was extracted from 10-15 ml fresh peripheral blood using a BloodGen Maxi Kit (Takara Biotechnology Co., Ltd., Dalian, China). Genomic DNA concentrations were adjusted to $50 \mathrm{ng} / \mu \mathrm{l}$ prior to genotyping. Samples were bar-coded to ensure accurate and reliable sample processing and storage.

Four SNPs (rs1126477, rs1126478, rs2073495 and rs9110) of the LTF gene are located in chromosome 3. These SNPs are located between positions 46480801 and 46501268 on the chromosome and each can result in a change in its associated amino acid residue. Genotyping of 20 genomic samples was performed at BGI Asia (Shenzhen, China) using MassARRAY technology according to a standard protocol (http://www.genomics.cn) $(3,15)$.

Protein extraction and digestion. The tissue samples of NPC and chronic inflammation of nasopharyngeal mucosa were lysed using a protein extraction buffer consisting of $50 \mathrm{mM}$ Tris ( $\mathrm{pH} 7.4), 150 \mathrm{mM} \mathrm{NaCl}, 1 \%$ Triton X-100, $1 \%$ sodium deoxycholate, $0.1 \%$ sodium dodecyl sulfate (SDS), sodium orthovanadate, sodium fluoride, ethylenediaminetetraacetic acid (EDTA) and leupeptin, supplemented with 1X Halt Protease Inhibitor Cocktail (CWBiotech, Beijing, China) and 1X Halt Phosphatase Inhibitor Cocktail (Shanghai Biological BestBio Bebo, Shanghai, China). The protein concentration was estimated using a Pierce ${ }^{\mathrm{TM}}$ bicinchoninic acid (BCA) assay (Thermo
Fisher Scientific, Inc., Carlsbad, CA, USA). Then, $50 \mu \mathrm{g}$ of each protein preparation was loaded on a $10 \%$ SDS-polyacrylamide gel electrophoresis (PAGE) gel. SDS-PAGE was run at $80 \mathrm{~V}$ for $40 \mathrm{~min}$, followed by $120 \mathrm{~V}$ for $90 \mathrm{~min}$. Protein bands were visualized using Coomassie brilliant blue G-250 (Sigma-Aldrich, St. Louis, MO, USA) and subsequently excised. The protein spots were de-stained reduced and alkylated. Next, trypsin (1.25 $\mu \mathrm{g} ; 1: 20$ enzyme/substrate ratio) was added to each band and in-gel digestion was performed at $37^{\circ} \mathrm{C}$ overnight $(\sim 16 \mathrm{~h})$. The generated peptides were extracted by sonication (15 min, ice cooling) of the gel pieces in $\sim 20 \mu \mathrm{l}$ of $50 \%$ acetonitrile (ACN; Merck Millipore, Darmstadt, Germany) in $0.1 \%$ formic acid (FA; Sigma-Aldrich, St. Louis, MO, USA) twice. Following extraction from the gel pieces, peptides were concentrated to dryness by centrifugation in SPD1010 SpeedVac (Themo Fisher Scientific, Inc.) and re-dissolved with $2 \% \mathrm{ACN}$ in $0.1 \%$ FA prior to liquid chromatography-tandem mass spectrometry (LC-MS/MS) analysis (16-18).

LC-MS/MS analysis of peptides. LC-MS/MS analyses were performed on an Ultimate 3000 RSLCnano system online coupled to an LTQ Orbitrap Velos Pro mass spectrometer (both Thermo Fisher Scientific, Inc., Bremen, Germany). Peptides were diluted with $0.1 \%$ FA and, for each analysis, $30 \mu \mathrm{l}$ of sample was injected. Following injection, peptides were pre-concentrated with $0.1 \%$ FA and $3 \%$ ACN on a trap column ( $\mu$-Precolumn C18 PepMap 100; $300 \mu \mathrm{m}$ x 5 mm, $5 \mu \mathrm{m}, 100 \AA$ А; Thermo Fisher Scientific, Inc.) at a flow rate of $300 \mathrm{nl} / \mathrm{min}$ for $5 \mathrm{~min}$. Subsequently, the analyte was transferred to the analytical column (Acclaim ${ }^{\circledR}$ PepMap RSLC; $75 \mu \mathrm{m}$ x $15 \mathrm{~cm}$, nano Viper, C18; $2 \mu \mathrm{m}, 100 \AA$ A Thermo Fisher Scientific, Inc.) and separated using a $120 \mathrm{~min}$ gradient from 5 to $40 \%$ solvent $\mathrm{B}$ at a flow rate of $300 \mathrm{nl} / \mathrm{min}$ (solvent $\mathrm{A}, 0.1 \%$ FA; solvent B, $0.08 \%$ FA, $80 \%$ ACN). The mass spectrometer was operated in a data-dependent mode. The general MS parameters were as follows: Spray voltage, $2.0 \mathrm{kV}$; capillary temperature, $275^{\circ} \mathrm{C}$. For data-dependent MS/MS analyses, the software XCalibur (Thermo Fisher Scientific, Inc.) was used. Full scan MS spectra were acquired at a mass resolution of 60,000 (mass range, 350-2,000 m/z) in the Orbitrap analyzer. For label-free analyses, tandem mass spectra of the ten most abundant peaks were acquired in the linear ion trap by peptide fragmentation using collision-induced dissociation. Normalized collision energy was set to $35 \%$ and an isolation width of $2 \mathrm{~m} / \mathrm{z}$ was selected (16-18).

Protein identification and quantification. Protein identification was performed with Proteome Discoverer 1.4 software (Themo Fisher Scientific, Inc.). Briefly, Thermo raw-files were imported and searched against the UniProtKB/Swiss-Prot database (release 2014_10; http://www.uniprot.org/). For database searches, mass tolerances were set to $10 \mathrm{ppm}$ and $0.8 \mathrm{Da}$ for precursor and fragment ions, respectively. Taxonomy was restricted to human, and one enzymatic miscleavage was allowed. For label-free analyses, modifications of cysteine (carbamidomethyl, static) and methionine (oxidation, variable) were considered. Confidence of peptide identifications was estimated using the percolator function, implemented in Proteome Discoverer. Instead of determining the peptide confidence based on a single metric-like Mascot ion score, the 
Table I. Characteristics of patients with nasopharyngeal carcinoma and chronic inflammation of nasopharyngeal mucosa.

\begin{tabular}{|c|c|c|c|c|c|c|}
\hline $\begin{array}{l}\text { Sample } \\
\text { ID }\end{array}$ & $\begin{array}{c}\text { WHO } \\
\text { histological diagnosis }\end{array}$ & $\begin{array}{l}\text { 'A-G-G-T' } \\
\text { haplotype }\end{array}$ & $\mathrm{TNM}^{\mathrm{b}}$ & Grade & Gender & $\begin{array}{c}\text { Age } \\
\text { (years) }\end{array}$ \\
\hline N1 & Chronic inflammation & With & - & - & Female & 45 \\
\hline $\mathrm{N} 2$ & Chronic inflammation & With & - & - & Female & 39 \\
\hline N3 & Chronic inflammation & Without & - & - & Male & 60 \\
\hline N4 & Chronic inflammation & With & - & - & Female & 64 \\
\hline N5 & Chronic inflammation & Without & - & - & Male & 55 \\
\hline N6 & Chronic inflammation & Without & - & - & Female & 38 \\
\hline N7 & Chronic inflammation & Without & - & - & Male & 57 \\
\hline N8 & Chronic inflammation & With & - & - & Female & 69 \\
\hline N9 & Chronic inflammation & Without & - & - & Male & 47 \\
\hline N10 & Chronic inflammation & With & - & - & Male & 48 \\
\hline N11 & Chronic inflammation & With & - & - & Female & 54 \\
\hline N12 & Chronic inflammation & With & - & - & Male & 62 \\
\hline N13 & Chronic inflammation & With & - & - & Female & 63 \\
\hline N14 & Chronic inflammation & Without & - & - & Male & 39 \\
\hline N15 & Chronic inflammation & With & - & - & Female & 45 \\
\hline N16 & Chronic inflammation & Without & - & - & Male & 52 \\
\hline N17 & Chronic inflammation & Without & - & - & Female & 57 \\
\hline N18 & Chronic inflammation & Without & - & - & Male & 61 \\
\hline N19 & Chronic inflammation & With & - & - & Male & 48 \\
\hline $\mathrm{N} 20$ & Chronic inflammation & Without & - & - & Female & 44 \\
\hline T01 & WHO II & Without & T1N0M0 & III & Male & 62 \\
\hline T02 & WHO II & Without & $\mathrm{T} 2 \mathrm{~N} 1 \mathrm{M} 0$ & II & Female & 41 \\
\hline T03 & WHO II & Without & $\mathrm{T} 4 \mathrm{~N} 2 \mathrm{M} 0$ & III & Male & 49 \\
\hline T04 & WHO II & With & T4N2M0 & I & Female & 51 \\
\hline T05 & WHO II & With & T4N0M0 & II & Male & 63 \\
\hline T06 & WHO II & Without & T1N0M0 & I & Female & 44 \\
\hline T07 & WHO II & With & $\mathrm{T} 1 \mathrm{~N} 3 \mathrm{M} 0$ & II & Male & 39 \\
\hline T08 & WHO II & Without & $\mathrm{T} 2 \mathrm{~N} 1 \mathrm{M} 0$ & I & Female & 57 \\
\hline T09 & WHO II & With & T1N1M0 & IV & Male & 66 \\
\hline $\mathrm{T} 10$ & WHO II & With & T1N1M0 & II & Male & 53 \\
\hline T11 & WHO II & Without & T1N0M0 & IV & Female & 48 \\
\hline $\mathrm{T} 12$ & WHO II & With & T4N0M0 & III & Male & 50 \\
\hline $\mathrm{T} 13$ & WHO II & Without & T1N1M0 & IV & Female & 37 \\
\hline $\mathrm{T} 14$ & WHO II & Without & T1N3M0 & II & Male & 59 \\
\hline $\mathrm{T} 15$ & WHO II & With & $\mathrm{T} 4 \mathrm{~N} 2 \mathrm{M} 0$ & III & Male & 67 \\
\hline T16 & WHO II & With & $\mathrm{T} 1 \mathrm{~N} 2 \mathrm{M} 0$ & I & Female & 46 \\
\hline $\mathrm{T} 17$ & WHO II & Without & T1N0M0 & IV & Male & 43 \\
\hline $\mathrm{T} 18$ & WHO II & With & T1N0M0 & IV & Male & 58 \\
\hline T19 & WHO II & Without & $\mathrm{T} 2 \mathrm{~N} 2 \mathrm{M} 0$ & I & Female & 52 \\
\hline $\mathrm{T} 20$ & WHO II & With & T3N1M0 & IV & Male & 41 \\
\hline
\end{tabular}

${ }^{\mathrm{a} C}$ Constructed with rs1126477, rs 1126478, rs2073495 and rs9110. ${ }^{\mathrm{b}} \mathrm{T}$ (tumor size), N (lymph node metastasis), and M (metastasis). WHO, World Health Organization; chronic inflammation, tissue classified as chronic inflammation of nasopharyngeal mucosa; WHO II, tissue classified as non-keratinizing carcinoma of nasopharyngeal type.

percolator was used as it discriminates correct from incorrect peptide spectrum matches based on multiple orthogonal score criteria, leading to accurate and sensitive peptide identifications. Peptide identifications with false discovery rates $\leq 1 \%$ (q-value $\leq 0.01$ ) were discarded.
Western blot analysis. Proteins from biopsy samples were prepared with lysis buffer [1\% Nonidet P-40; $50 \mathrm{mM}$ Tris- $\mathrm{HCl}$, pH 7.5; 50 mM NaF; 2 mM EDTA; $10 \%$ glycerol; plus complete protease inhibitor mixture (Roche Diagnostics, Indianapolis, USA) with $\mathrm{NaCl}$ adjusted to $400 \mathrm{mM}$ ]. The protein 
Table II. Differentially expressed genes in NPC tissues between those with and those without the 'A-G-G-T' haplotype.

\begin{tabular}{|c|c|c|c|c|c|c|c|c|}
\hline \multirow[b]{2}{*}{ Rank } & \multicolumn{4}{|c|}{ NPC tissues with 'A-G-G-T' haplotype } & \multicolumn{4}{|c|}{ NPC tissues without 'A-G-G-T' haplotype } \\
\hline & Gene $^{a}$ & $\begin{array}{c}\text { Unique } \\
\text { peptides }^{\mathrm{b}}\end{array}$ & $\begin{array}{c}\text { Coverage }^{c} \\
(\%)\end{array}$ & $\begin{array}{l}\text { Protein } \\
\text { score }^{\mathrm{d}}\end{array}$ & Gene $^{a}$ & $\begin{array}{l}\text { Unique } \\
\text { peptides }^{\mathrm{b}}\end{array}$ & $\begin{array}{c}\text { Coverage } e^{c} \\
(\%)\end{array}$ & $\begin{array}{r}\text { Protein } \\
\text { score }^{\mathrm{d}}\end{array}$ \\
\hline 1 & SARNP & 20 & 60.18 & 201.61 & RNF44 & 27 & 42.47 & 187.91 \\
\hline 2 & TLE2 & 19 & 59.17 & 270.20 & JNK2 & 27 & 42.24 & 182.95 \\
\hline 3 & DECR1 & 17 & 49.40 & 175.15 & GNPDA1 & 22 & 33.22 & 156.63 \\
\hline 4 & RNF207 & 16 & 26.03 & 159.53 & WARS2 & 19 & 41.31 & 130.17 \\
\hline 5 & EXOSC2 & 16 & 46.82 & 90.21 & PACSIN2 & 18 & 32.57 & 123.24 \\
\hline 6 & IGSF22 & 15 & 52.50 & 182.41 & PGK1 & 16 & 27.93 & 122.51 \\
\hline 7 & PMFBP1 & 14 & 45.16 & 158.00 & MITD1 & 16 & 47.62 & 108.82 \\
\hline 8 & PSMG2 & 14 & 34.12 & 186.88 & MTX2 & 16 & 48.98 & 91.49 \\
\hline 9 & ZNF469 & 14 & 47.57 & 138.74 & DPM1 & 15 & 25.80 & 78.52 \\
\hline 10 & UCK2 & 14 & 43.85 & 69.90 & MTCH2 & 14 & 24.75 & 74.95 \\
\hline 11 & EXOSC3 & 13 & 51.69 & 75.30 & DERA & 13 & 54.35 & 70.71 \\
\hline 12 & STK36 & 13 & 45.51 & 65.20 & SUGT1 & 13 & 39.45 & 66.02 \\
\hline 13 & PNPO & 12 & 50.16 & 120.21 & RFC5 & 11 & 52.43 & 64.83 \\
\hline 14 & CALCOCO1 & 12 & 46.42 & 100.79 & SYNE1 & 11 & 35.27 & 63.10 \\
\hline 15 & SYPL1 & 12 & 62.67 & 89.77 & GSR & 10 & 36.84 & 61.74 \\
\hline 16 & MAPK8IP3 & 12 & 54.73 & 69.40 & ZNF106 & 10 & 57.72 & 61.35 \\
\hline 17 & ATP8A1 & 12 & 55.36 & 110.40 & SCO1 & 10 & 38.00 & 59.86 \\
\hline 18 & EEF1D & 12 & 56.22 & 158.47 & PSMA4 & 10 & 42.16 & 55.18 \\
\hline 19 & UTP11L & 12 & 47.73 & 122.66 & GNB1 & 10 & 23.99 & 54.65 \\
\hline 20 & CAPRIN1 & 12 & 44.49 & 91.04 & CIP29 & 10 & 23.73 & 52.38 \\
\hline
\end{tabular}

${ }^{a}$ Top 20 proteins, ranked by number of unique peptides. ${ }^{b}$ Unique peptides represent the number of peptide sequences that are unique to a protein group. ${ }^{\mathrm{c}}$ Coverage displays by default the percentage of the protein sequence that is covered by the identified peptides. ${ }^{\mathrm{d} P r o t e i n}$ score is automatically generated by the instrument (linear trap quadrupole Orbitrap Velos Pro mass spectrometer; Thermo Fisher Scientific, Inc.). NPC, nasopharyngeal carcinoma.

concentrations were determined using a Pierce ${ }^{\mathrm{TM}}$ BCA protein assay. Extracts containing $50 \mu \mathrm{g}$ of proteins were separated on $10 \%$ SDS-PAGE gels and electroblotted onto Hyclone ${ }^{\mathrm{TM}}$ nitrocellulose membranes (GE Healthcare Life Sciences, Logan, UT, USA). The membranes were blocked using Tris-buffered saline/Tween $20(25 \mathrm{mM}$ Tris-HCl; $150 \mathrm{mM}$ $\mathrm{NaCl}, \mathrm{pH} 7.5 ; 0.05 \%$ Tween 20 ) containing 5\% non-fat milk, followed by overnight incubation at $4{ }^{\circ} \mathrm{C}$ with primary antibodies against the $\mathrm{N}$-terminus of human c-Jun $\mathrm{N}$-terminal kinase 2 [JNK2; rabbit polyclonal immunoglobulin $(\mathrm{Ig}) \mathrm{G}$; dilution, 1:500; catalogue number sc-827; Santa Cruz Biotechnology, Inc., Santa Cruz, Dallas, TX, USA] and against full-length human GAPDH (mouse monoclonal IgG1; dilution, 1:3,000; catalogue number sc-365062; Santa Cruz Biotechnology, Inc.). Following three washes, goat anti-rabbit IgG horseradish peroxidase-conjugated secondary antibody (dilution, 1:5,000; catalogue number sc-2030; Santa Cruz Biotechnology,Inc.) were added, and incubated for $1 \mathrm{~h}$. The signals were visualized using an enhanced chemiluminescence detection system (Universal Hood II; Bio-Rad Laboratories, Inc., Hercules, CA, USA) with Image Lab $^{\mathrm{TM}}$ software version 2.0 (Bio-Rad Laboratories, Inc.).

miRNA microarray assay. Microarray assays of NPC tissues and non-tumor nasopharyngeal epithelium tissues miRNAs were outsourced to Shanghai OE Biotech Co., Ltd. (Shanghai, China). To enrich global miRNA, total RNA extract was purified using Ambion ${ }^{\circledR}$ mirVana ${ }^{\mathrm{TM}}$ miRNA Isolation Kit (Thermo Fisher Scientific, Inc., Carlsbad, CA, USA), and then labeled and hybridized. Briefly, 100 ng of miRNA was labeled using the Agilent miRNA Complete Labeling and Hybridization Kit (Agilent Technologies, Inc., Santa Clara, CA, USA) according to the manufacturer's instructions. The labeled RNA was hybridized to the Agilent human miRNA microarray, which contains probes for 1,205 human miRNAs and 144 human viral miRNAs from the Sanger database (version 16.0) according to the manufacturer's instructions. Arrays were scanned with the Affymetrix GeneChip ${ }^{\circledR}$ Scanner 3000 (Agilent Technologies, Inc.), and the raw data were normalized and analyzed by GeneSpring GX software version 7.3 (Agilent Technologies, Inc.). The GeneSpring software generated an average value for each miRNA from the repeated probes. Microarray assay was performed in triplicates, utilizing three independent sets of RNA preparations. miRNA signal intensities were $\log _{2}$ transformed, and analyzed for differentially expressed miRNAs by using the Significance Analysis of Microarrays (version 3.01), and the P-values of the $t$-test were calculated. Differentially detected miRNA signals with $\geq 1.5$ fold-change and $\mathrm{P}<0.05$ were considered statistically significant. Differentially 
Table III. Differentially expressed genes in non-tumor nasopharyngeal epithelium tissues between those with and those without the 'A-G-G-T' haplotype.

\begin{tabular}{|c|c|c|c|c|c|c|c|c|}
\hline \multirow[b]{2}{*}{ Rank } & \multicolumn{4}{|c|}{$\begin{array}{l}\text { Non-tumor nasopharyngeal epithelium } \\
\text { tissues with 'A-G-G-T' haplotype }\end{array}$} & \multicolumn{4}{|c|}{$\begin{array}{l}\text { Non-tumor nasopharyngeal epithelium } \\
\text { tissues without 'A-G-G-T' haplotype }\end{array}$} \\
\hline & Gene $^{a}$ & $\begin{array}{l}\text { Unique } \\
\text { peptides }^{b}\end{array}$ & $\begin{array}{l}\text { Coverage }{ }^{c} \\
(\%)\end{array}$ & $\begin{array}{l}\text { Protein } \\
\text { score }^{\mathrm{d}}\end{array}$ & Gene $^{a}$ & $\begin{array}{l}\text { Unique } \\
\text { peptides }^{\mathrm{b}}\end{array}$ & $\begin{array}{l}\text { Coverage }^{c} \\
(\%)\end{array}$ & $\begin{array}{l}\text { Protein } \\
\text { score }^{\mathrm{d}}\end{array}$ \\
\hline 1 & PRPF4B & 19 & 59.29 & 135.21 & HNRNPAB & 32 & 56.05 & 147.23 \\
\hline 2 & PSME2 & 19 & 54.44 & 78.39 & RALYL & 25 & 48.49 & 81.92 \\
\hline 3 & WDR61 & 16 & 37.95 & 60.94 & FAM49B & 22 & 42.81 & 70.34 \\
\hline 4 & ECI1 & 12 & 42.81 & 64.96 & GNB2 & 22 & 37.01 & 68.36 \\
\hline 5 & FLNB & 11 & 22.95 & 43.52 & MLEC & 21 & 52.37 & 76.00 \\
\hline 6 & SNRPA & 11 & 58.23 & 61.73 & AK2 & 21 & 16.10 & 64.23 \\
\hline 7 & PSMA7 & 9 & 34.03 & 56.65 & NIT2 & 20 & 40.07 & 53.41 \\
\hline 8 & NUDT21 & 8 & 15.96 & 62.85 & PNP & 19 & 19.72 & 62.36 \\
\hline 9 & EIF3J & 7 & 41.91 & 51.31 & TTLL5 & 17 & 49.00 & 77.06 \\
\hline 10 & PSMG1 & 6 & 51.79 & 51.16 & HNRNPC & 15 & 37.57 & 49.18 \\
\hline 11 & PNP & 6 & 24.07 & 60.41 & EFHD2 & 15 & 44.64 & 58.63 \\
\hline 12 & HPRT1 & 6 & 39.70 & 45.02 & JNK2 & 14 & 40.18 & 47.68 \\
\hline 13 & ANXA4 & 5 & 43.44 & 44.42 & SNRPB2 & 13 & 20.34 & 66.65 \\
\hline 14 & PRPS1L1 & 5 & 37.15 & 41.81 & PSMA4 & 13 & 55.56 & 45.61 \\
\hline 15 & HSPH1 & 5 & 36.07 & 40.28 & HIBADH & 13 & 28.03 & 54.89 \\
\hline 16 & FUBP1 & 5 & 36.59 & 37.52 & TSN & 12 & 48.63 & 53.78 \\
\hline 17 & ATP5C1 & 4 & 42.97 & 46.49 & CTSD & 12 & 36.29 & 42.85 \\
\hline 18 & SUCLG1 & 4 & 37.65 & 35.93 & ATP1B3 & 12 & 38.16 & 62.46 \\
\hline 19 & YWHAH & 4 & 31.50 & 55.79 & PSME3 & 11 & 37.55 & 51.43 \\
\hline 20 & HSD17B10 & 4 & 35.92 & 44.11 & PSMD8 & 11 & 41.91 & 41.26 \\
\hline
\end{tabular}

${ }^{a}$ Top 20 proteins, ranked by number of unique peptides. ${ }^{b}$ Unique peptides represent the number of peptide sequences that are unique to a protein group. ${ }^{~ C}$ Coverage displays by default the percentage of the protein sequence that is covered by the identified peptides. ${ }^{\mathrm{d} P r o t e i n}$ score is automatically generated by the instrument (linear trap quadrupole Orbitrap Velos Pro mass spectrometer; Thermo Fisher Scientific, Inc.).

expressed miRNAs detected in the triplicate experiments were selected for further analysis.

Validation of differential miRNAs. To validate the reliability of microarray data, reverse transcription (RT)-quantitative polymerase chain reaction (qPCR) was performed to detect the levels of hsa-miR-1256, hsa-miR-659 and hsa-miR-298 in NPC and non-tumor nasopharyngeal epithelium tissues with and without the 'A-G-G-T' haplotype. For miRNA RT-qPCR, $2 \mu \mathrm{g}$ of total RNA was extracted from NPC and non-tumor nasopharyngeal epithelium tissues with TRIzol reagent (Qiagen GmbH, Hilden, Germany), and reverse transcribed into cDNA with an RT kit according to the manufacturer's instructions (Promega Corporation, Madison, WI, USA), using miRNA specific primers (hsa-miR-1256, hsa-miR-659 and hsa-miR-298; Bulge-Loop ${ }^{\mathrm{TM}}$ miRNA qPCR primers), which were synthesized by Guangzhou RiboBio Co., Ltd. (Guangzhou, China). The RT products were amplified by qPCR in a CFX Connect Real-Time PCR Detection System (Bio-Rad Laboratories, Inc.) using the miScript SYBR Green PCR kit (Qiagen $\mathrm{GmbH}$ ) according to the manufacturer's instructions. The cycling conditions were as follows: $95^{\circ} \mathrm{C}$ for $10 \mathrm{~min}$, followed by 40 cycles of $95^{\circ} \mathrm{C}$ for $15 \mathrm{sec}$ and $60^{\circ} \mathrm{C}$ for $1 \mathrm{~min}$. U6 was used as the internal control. The relative expression was calculated based on the comparative quantification cycle method (19) using Bio-Rad CFK manager software version 2.0 (Bio-Rad Laboratories, Inc.). The experiments were repeated three times.

Statistical analysis. Distribution of age/gender was compared across cases by status using $\chi^{2}$ tests. Haplotyper (http://www. people.fas.harvard.edu/ junliu/Haplo/click.html) software was used for haplotype inference (20-22). Differences in quantitative variables between groups were analyzed by the Student's $t$-test using SPSS version 13.0 (SPSS, Inc., Chicago, IL, USA). $\mathrm{P}<0.05$ was considered to indicate statistically significant differences.

\section{Results}

Identification of differentially expressed proteins in NPC tissues between those with and without the 'A-G-G-T' haplotype. To identify the differentially expressed proteins in NPC tissues between those with and without the 'A-G-G-T' haplotype, the protein profiles of NPC tissues were constructed using LTQ Orbitrap technology. This revealed 176 proteins 
Table IV. MicroRNAs differentially expressed in NPC tissues between those with and those without the 'A-G-G-T' haplotype.

\begin{tabular}{|c|c|c|c|c|}
\hline \multirow[b]{2}{*}{ Rank } & \multicolumn{2}{|c|}{ Downregulated microRNA ${ }^{a}$} & \multicolumn{2}{|c|}{ Upregulated microRNA $^{\mathrm{a}}$} \\
\hline & Systematic name & Fold change & Systematic name & Fold change \\
\hline 1 & hsa-miR-720 & 36.09 & hsa-miR-17 & 34.09 \\
\hline 2 & hsa-miR-218 & 28.19 & hsa-miR-29b & 32.56 \\
\hline 3 & hsa-miR-1274b & 28.05 & hsa-miR-1256 & 30.26 \\
\hline 4 & hsa-miR-205 & 25.20 & hsa-miR-23a & 29.44 \\
\hline 5 & hsa-miR-221 & 22.22 & hsa-miR-21 & 25.53 \\
\hline 6 & hsa-miR-2276 & 21.15 & hsa-miR-20a & 24.54 \\
\hline 7 & hsa-miR-2355-5p & 20.69 & hsa-miR-103 & 24.14 \\
\hline 8 & hsa-miR-19b & 20.44 & hsa-miR-15b & 23.95 \\
\hline 9 & hsa-miR-20a & 19.76 & hsa-miR-22 & 22.47 \\
\hline 10 & hsa-miR-16 & 18.06 & hsa-miR-27a & 21.74 \\
\hline 11 & hsa-miR-24 & 18.05 & hsa-miR-100 & 20.63 \\
\hline 12 & hsa-miR-2909 & 17.24 & hsa-miR-27b & 19.25 \\
\hline 13 & hsa-miR-205 & 17.24 & hsa-miR-200b & 18.12 \\
\hline 14 & hsa-miR-1260 & 16.59 & hsa-miR-659 & 16.89 \\
\hline 15 & hsa-miR-296-5p & 15.66 & hsa-miR-494 & 16.39 \\
\hline 16 & hsa-miR-4286 & 15.49 & hsa-miR-29a & 16.29 \\
\hline 17 & hsa-miR-1260b & 13.84 & hsa-miR-31 & 14.53 \\
\hline 18 & hsa-miR-24 & 13.49 & hsa-miR-141 & 13.23 \\
\hline 19 & hsa-miR-200c & 13.16 & hsa-miR-19a & 13.08 \\
\hline 20 & hsa-miR-4284 & 12.80 & hsa-miR-320d & 13.04 \\
\hline
\end{tabular}

aWith 'A-G-G-T' haplotype vs. without 'A-G-G-T' haplotype.

that were only present in NPC tissues with the 'A-G-G-T' haplotype, and 154 proteins that only appeared in NPC tissues without the 'A-G-G-T' haplotype. According to the frequency of unique peptides, SAP domain-containing ribonucleoprotein was the highest in NPC tissues with the 'A-G-G-T' haplotype, followed by transducin-like enhancer of split 2, and 2,4-dienoyl CoA reductase 1 mitochondrial. Ring finger protein 44 was the highest in NPC tissues without the 'A-G-G-T' haplotype, and the second was JNK2. Full details of the top 20 proteins in NPC tissues with/without the 'A-G-G-T' haplotype are presented in Table II.

Identification of differentially expressed proteins in non-tumor nasopharyngeal epithelium tissues between those with and without the 'A-G-G-T' haplotype. To explore the different proteins in non-tumor nasopharyngeal epithelium tissues according to the presence of the 'A-G-G-T' haplotype, the protein profiles of non-tumor nasopharyngeal epithelium tissues were constructed as described. This identified 162 proteins that were only present in non-tumor nasopharyngeal epithelium tissues with the 'A-G-G-T' haplotype, and 180 proteins that only appeared in non-tumor nasopharyngeal epithelium tissues without the 'A-G-G-T' haplotype. According to the frequency of unique peptides, pre-mRNA processing factor $4 \mathrm{~B}$ was the highest in non-tumor nasopharyngeal epithelium tissue with the 'A-G-G-T' haplotype, followed by proteasome (prosome, macropain) activator subunit 2 (PA28 $\beta$ ), WD repeat domain 61, and enoyl-CoA delta isomerase 1. Heterogeneous nuclear ribonucleoprotein $\mathrm{A} / \mathrm{B}$ was the highest in non-tumor nasopharyngeal epithelium tissue without the 'A-G-G-T' haplotype. The JNK2 protein was also in the list of the top 20 different proteins in tissues without the 'A-G-G-T' haplotype. Full details of the top 20 proteins in non-tumor nasopharyngeal epithelium tissues with/without the 'A-G-G-T' haplotype are presented in Table III. Notably, JNK2 protein was present in NPC tissue and non-tumor nasopharyngeal epithelium tissues without the 'A-G-G-T' haplotype.

Validation of JNK2 protein by western blotting. Through LTQ Orbitrap technology, JNK2 protein was found to be present in NPC tissue and non-tumor nasopharyngeal epithelium tissues without the 'A-G-G-T' haplotype. This suggested that the expression levels of JNK2 may be associated with polymorphic $L T F$ haplotypes in human NPC. To verify whether JNK2 was upregulated in NPC tissue and non-tumor nasopharyngeal epithelium tissues without the 'A-G-G-T' haplotype, the expression level of JNK2 was assessed in 4 NPC samples and 4 non-tumor nasopharyngeal epithelium samples by western blotting. Whether in the NPC tissues or non-tumor nasopharyngeal epithelium tissues, JNK2 was higher in tissues without the 'A-G-G-T' haplotype than with the 'A-G-G-T' haplotype. However, the overall expression level of JNK2 was lower in non-tumor nasopharyngeal epithelium tissues than NPC tissues (Fig. 1). This confirmed the reliability of the results obtained by 
Table V. MicroRNAs differentially expressed in non-tumor nasopharyngeal epithelium tissues between those with and those without the 'A-G-G-T' haplotype.

\begin{tabular}{|c|c|c|c|c|}
\hline \multirow[b]{2}{*}{ Rank } & \multicolumn{2}{|c|}{ Downregulated microRNA ${ }^{a}$} & \multicolumn{2}{|c|}{ Upregulated microRNA $^{\mathrm{a}}$} \\
\hline & Systematic name & Fold change & Systematic name & Fold change \\
\hline 1 & hsa-miR-18a-5p & 72.94 & hsa-miR-652-3p & 69.10 \\
\hline 2 & hsa-miR-29b-3p & 50.51 & hsa-miR-664-3p & 58.78 \\
\hline 3 & hsa-miR-1249 & 40.58 & hsa-miR-24-1-5p & 58.10 \\
\hline 4 & hsa-miR-27a & 39.70 & hsa-miR-298 & 48.01 \\
\hline 5 & hsa-miR-185-5p & 36.31 & hsa-miR-3646 & 47.66 \\
\hline 6 & hsa-miR-28-5p & 36.26 & hsa-miR-223 & 37.53 \\
\hline 7 & hsa-miR-3907 & 36.03 & hsa-miR-3125 & 37.24 \\
\hline 8 & hsa-miR-483-3p & 35.38 & hsa-miR-660-5p & 37.23 \\
\hline 9 & hsa-miR-1288 & 34.73 & hsa-miR-128 & 36.88 \\
\hline 10 & hsa-miR-16-2-3p & 31.73 & hsa-miR-744-5p & 34.01 \\
\hline 11 & hsa-miR-877-3p & 25.88 & hsa-miR-582-5p & 30.05 \\
\hline 12 & hsa-miR-19b-1-5p & 25.07 & hsa-miR-26a & 28.69 \\
\hline 13 & hsa-miR-27b & 24.83 & hsa-miR-1470 & 28.59 \\
\hline 14 & hsa-miR-132-3p & 24.78 & hsa-miR-140-5p & 28.41 \\
\hline 15 & hsa-miR-542-5p & 23.61 & hsa-miR-374b-5p & 28.34 \\
\hline 16 & hsa-miR-2861 & 23.16 & hsa-miR-3197 & 23.33 \\
\hline 17 & hsa-miR-296-5p & 22.51 & hsa-miR-23b & 22.53 \\
\hline 18 & hsa-miR-3907 & 22.22 & hsa-miR-192-5p & 22.47 \\
\hline 19 & hsa-miR-362-5p & 28.72 & hsa-miR-99a-5p & 22.47 \\
\hline 20 & hsa-miR-505-3p & 28.24 & hsa-miR-205-3p & 22.40 \\
\hline
\end{tabular}

a'With 'A-G-G-T' haplotype vs. without 'A-G-G-T' haplotype.
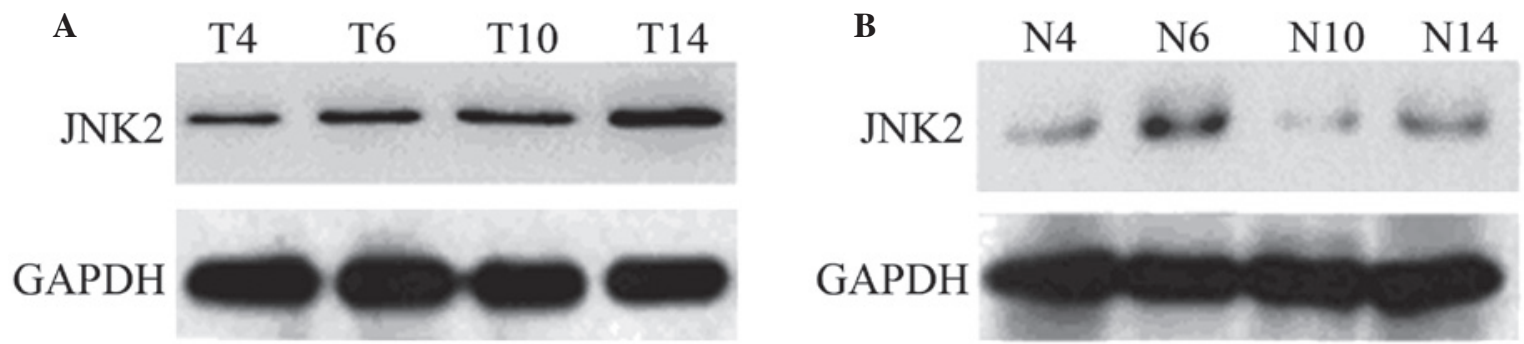

Figure 1. Effect of 'A-G-G-T' haplotype on JNK2 protein expression levels assessed by western blot analysis. (A) NPC tissues and (B) non-tumor nasopharyngeal epithelium tissues. T4, T10, N4 and N10 indicate samples from individuals with the 'A-G-G-T' haplotype; T6, T14, N6 and N14 indicate individuals without the 'A-G-G-T' haplotype.

LTQ Orbitrap technology, and confirmed that JNK2 was higher in tissues without the 'A-G-G-T' haplotype than with the 'A-G-G-T' haplotype.

miRNAs differentially expressed in NPC tissues between those with and without the 'A-G-G-T' haplotype. miRNA microarray analysis was conducted to investigate the differentially expressed miRNA profiles of NPC tissues with/without the 'A-G-G-T' haplotype. This analysis identified 104 miRNAs that had lower expression in NPC tissue with the 'A-G-G-T' haplotype and 83 miRNAs that had higher expression in NPC tissue with the 'A-G-G-T' haplotype. According to the fold change, hsa-miR-720 had the highest downregulated fold change in NPC tissue with the 'A-G-G-T' haplotype, followed by hsa-miR-218 and hsa-miR-1274b (Table IV). However, hsa-miR-17 had the highest upregulated fold change in NPC tissue without the 'A-G-G-T' haplotype, followed by hsa-miR-29b and hsa-miR-1256. Details of the top 20 miRNAs are presented in Table IV.

The top 20 miRNAs that were dysregulated in NPC were further analyzed to predict their target genes; hsa-miR-1256 and hsa-miR-659, which were downregulated in NPC tissues without the 'A-G-G-T' haplotype, are potentially targeted to the $J N K 2$ gene.

miRNAs differentially expressed in non-tumor nasopharyngeal epithelium tissues between those with and without the 'A-G-G-T' haplotype. miRNA microarray analysis of 
A

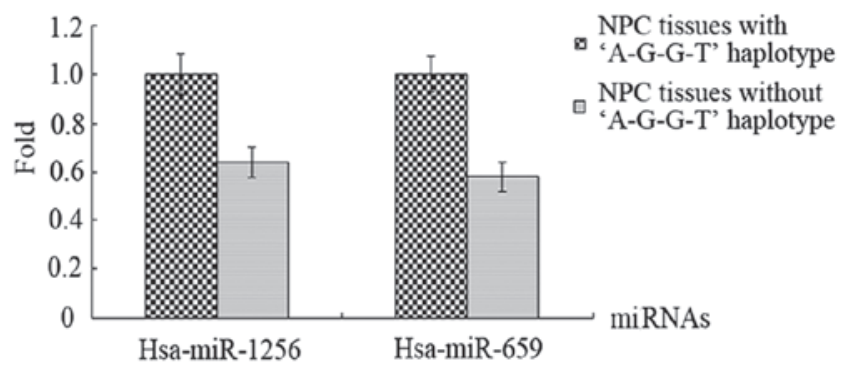

B



Figure 2. Validation of hsa-miR-1256, hsa-miR-659 and hsa-miR-298 by reverse transcription-quantitative polymerase chain reaction analysis. (A) Validation of hsa-miR-1256 and hsa-miR-659 in NPC tissues with/without the 'A-G-G-T' haplotype. (B) Validation of hsa-miR-298 in non-tumor nasopharyngeal epithelium tissues with/without the 'A-G-G-T' haplotype.

non-tumor nasopharyngeal epithelium tissues with/without the 'A-G-G-T' haplotype was conducted. This revealed that, in non-tumor nasopharyngeal epithelium tissue with the 'A-G-G-T' haplotype, 79 miRNAs had lower expression and 137 miRNAs had higher expression. According to the fold change, hsa-miR-18a-5p had the highest fold downregulation in non-tumor nasopharyngeal epithelium tissues with the 'A-G-G-T' haplotype, followed by hsa-miR-29b-3p and hsa-miR-1249. However, hsa-miR-652-3p had the highest fold upregulation in non-tumor nasopharyngeal epithelium tissue without the 'A-G-G-T' haplotype, followed by hsa-miR-664-3p, hsa-miR-24-1-5p and hsa-miR-298. Details of the top 20 miRNAs are presented in Table V.

The top 20 miRNAs dysregulated in non-tumor nasopharyngeal epithelium tissue were further analyzed to predict their target genes, and this revealed that hsa-miR-298 was downregulated in non-tumor nasopharyngeal epithelium tissue without the 'A-G-G-T' haplotype and is potentially targeted to the $J N K 2$ gene.

Validation of hsa-miR-1256, hsa-miR-659 and hsa-miR-298. The results of the differential protein profiles indicated that the expression levels of JNK2 may be associated with polymorphic LTF haplotypes in human NPC. The results of miRNA microarray analyses revealed that hsa-miR-1256, hsa-miR-659 and hsa-miR-298, which are potentially targeted to the JNK2 gene, were downregulated in NPC/non-tumor nasopharyngeal epithelium tissues without the 'A-G-G-T' haplotype (Fig. 2). Thus, these miRNAs (hsa-miR-1256, hsa-miR-659, and hsa-miR-298) were selected for RT-qPCR evaluation in 12 NPC tissues and 12 non-tumor nasopharyngeal epithelium tissues. The results revealed that hsa-miR-1256 and hsa-miR-659 were downregulated in NPC tissues without the 'A-G-G-T' haplotype compared with tissues with the 'A-G-G-T' haplotype; the fold changes were $0.64(\mathrm{P}=0.027)$ and $0.58(\mathrm{P}=0.031)$, respectively. Hsa-miR-298 was 0.61-fold downregulated in non-tumor nasopharyngeal epithelium tissues without the 'A-G-G-T' haplotype relative to tissues with the 'A-G-G-T' haplotype $(\mathrm{P}=0.019)$.

\section{Discussion}

LTF protein is a ubiquitous and abundant component of human exocrine secretions. As a member of the transferrin family, LTF is an iron-binding glycoprotein, and has been demonstrated to have multiple physiological functions. LTF is also present in specific granules of neutrophils (23-28). Its functions include roles in the immune response and the regulation of bone metabolism. LTF also sequesters essential iron to inhibit bacterial growth, as well as exhibiting non-iron-dependent antibacterial, antitumor, antiviral, antifungal, anti-inflammatory and immunoregulatory activities (23-28).

In the current study, JNK2 protein was determined to be upregulated in NPC tissues and non-tumor nasopharyngeal epithelium tissues without the 'A-G-G-T' haplotype. This suggested that the expression levels of JNK2 may be associated with polymorphic LTF haplotypes in human NPC. Western blot analyses confirmed the reliability of the results obtained by LTQ Orbitrap technology, and verified that JNK2 was higher in the NPC/non-tumor nasopharyngeal epithelium tissues without the 'A-G-G-T' haplotype than with the 'A-G-G-T' haplotype. Huang et al (29) reported that NAG7 promoted human NPC invasion and had a potential role in promoting NPC invasion via the regulation of estrogen receptor $\alpha$ and the H-Ras/p-c-Raf and JNK2/activator protein 1 /matrix metalloproteinase 1 signaling pathways. Raciti et al (30) reported that JNK2 prevented the accumulation of the acidic compartment in U937 cells undergoing autophagic flux and contributed to the survival of stressed cells (30). JNK1/2 deficiency has also been shown to increase branching morphogenesis and lead to defects in the clearance of lumenal epithelial cells. In the context of breast cancer development, deficiencies in JNK1/2 led to a significant increase in tumor formation (31). Radovich et al (32) reported that haplotypes in the vascular endothelial growth factor (VEGF) gene affected its gene expression. Furthermore, our previous study demonstrated that LTF inhibited the levels of c-Jun, c-Fos and JNK2 expression in NPC cell lines (4). Collectively, this evidence indicates that JNK2 may serve an important role in malignancy and that LTF may affect the expression of JNK2.

miRNAs (miRNAs) are a class of small non-coding RNAs that are non-randomly distributed in the genome, and alterations in the expression of these molecules have been reported in multiple types of cancer, including NPC (33-35). The present study aimed to investigate whether the miRNAs that potentially target $J N K 2$ have altered expression in NPC/non-tumor nasopharyngeal epithelium tissues without the 'A-G-G-T' haplotype. miRNA microarray analyses of NPC/non-tumor nasopharyngeal epithelium tissues 
with/without the 'A-G-G-T' haplotype were performed. The results revealed that hsa-miR-1256 and hsa-miR-659, which potentially target the $J N K 2$ gene, were downregulated in NPC tissues without the 'A-G-G-T' haplotype relative to those with the 'A-G-G-T' haplotype, with fold changes of 0.64 and 0.58 , respectively. Hsa-miR-298, another miRNA potentially targeting $J N K 2$, was 0.61 -fold downregulated in non-tumor nasopharyngeal epithelium tissues without the 'A-G-G-T' haplotype relative to those with the 'A-G-G-T' haplotype. Werk et al (36) found a differential suppression of ATP-binding cassette C 2 by miR-379 was caused by haplotype-dependent differences in mRNA secondary structures, resulting in changes in mRNA target accessibility or mRNA stability. A study by Koelsch et al (37) supported the notion that variants within the methyl $\mathrm{CpG}$ binding protein 2 gene could alter DNA methylation in other genetic loci, including the human leukocyte antigen and interferon-regulated genes. Li et al (38) found that isoflavone could increase the levels of miR-29a and miR-1256 and decrease expression of tripartite motif-containing 68 and phosphoglycerate kinase 1, which was mechanistically linked with inhibition of prostate cancer cell growth and invasion. The result of the study by Bao et al (39) suggested that miR-298 directly modulated multidrug resistance protein 1 (P-glycoprotein) expression and was associated with the chemoresistant mechanisms of metastatic human breast cancer.

In the present study, JNK2 protein was confirmed to be upregulated in NPC and non-tumor nasopharyngeal epithelium tissues without the 'A-G-G-T' haplotype by western blotting. In addition, hsa-miR-1256, hsa-miR-659 and hsa-miR-298 were predicted to be targets of the JNK2 gene, and the data confirmed that hsa-miR-1256 and hsa-miR-659 were downregulated in NPC tissues without the 'A-G-G-T' haplotype, compared with tissues with the 'A-G-G-T' haplotype, while hsa-miR-298 was 0.61-fold downregulated in non-tumor nasopharyngeal epithelium tissues without the 'A-G-G-T' haplotype, compared with tissues with the 'A-G-G-T' haplotype. Furthermore, the current miRNA analysis results also confirmed that JNK2 protein was upregulated in NPC and non-tumor nasopharyngeal epithelium tissues without the 'A-G-G-T' haplotype.

In conclusion, the present study demonstrated that the expression levels of JNK2 are associated with polymorphic LTF haplotypes in human NPC. However, further studies are required to investigate the mechanism of the association between LTF polymorphisms and expression level of JNK2 in normal nasopharyngeal epithelial tissues, and the pathological implications in the development of NPC.

\section{Acknowledgements}

This work was supported by National Natural Science Foundation of China (nos. 81272975, 81172302 and 81402270); the Key Project of Hunan Provincial Natural Science Foundation (no. 12JJ2044); the Project of Hunan Provincial Natural Science Foundation (no. 12JJ3121); Project of Hunan Provincial Development and Reform Commission; the Planned Science and Technology Project of Hunan Province (nos. 2010FJ3088, 2012FJ2014); and the Open-End Fund for the Valuable and Precision Instruments of Central South University.

\section{References}

1. Tuccari $\mathrm{G}$ and Barresi G: Lactoferrin in human tumours: Immunohistochemical investigations during more than 25 years. Biometals 24: 775-84, 2011.

2. Deng M, Zhang W, Tang H, Ye Q, Liao Q, Zhou Y, Wu M, Xiong W, Zheng Y, Guo X, et al: Lactotransferrin acts as a tumor suppressor in nasopharyngeal carcinoma by repressing AKT through multiple mechanisms. Oncogene 32: 4273-4283, 2013.

3. Zhou Y, Wang W, Zheng D, Peng S, Xiong W, Ma J, Zeng Z, Wu M, Zhou M, Xiang J, et al: Risk of nasopharyngeal carcinoma associated with polymorphic lactotransferrin haplotypes. Med Oncol 29: 1452-1462, 2012.

4. Zhou Y, Zeng Z, Zhang W, Xiong W, Wu M, Tan Y, Yi W, Xiao L, Li X, Huang C, et al: Lactotransferrin: A candidate tumor suppressor-deficient expression in human nasopharyngeal carcinoma and inhibition of NPC cell proliferation by modulating the mitogen-activated protein kinase pathway. Int J Cancer 123: 2065-2072, 2008.

5. Zhou Y, Zeng Z, Zhang W, Xiong W, Li X, Zhang B, Yi W, Xiao L, Wu M, Shen S, et al: Identification of candidate molecular markers of nasopharyngeal carcinoma by microarray analysis of subtracted cDNA libraries constructed by suppression subtractive hybridization. Eur J Cancer Prev 17: 561-571, 2008.

6. Ye Q, Zheng Y, Fan S, Qin Z, Li N, Tang A, Ai F, Zhang X, Bian Y, Dang W, et al: Lactoferrin deficiency promotes colitis-associated colorectal dysplasia in mice. PLoS One 9: e103298, 2014

7. Teng CT and Gladwell W: Single nucleotide polymorphisms (SNPs) in human lactoferrin gene. Biochem Cell Biol 84: 381-384, 2006.

8. Azevedo LF, Pecharki GD, Brancher JA, Cordeiro CA Jr, Medeiros KG, Antunes AA, Arruda ES, Werneck RI, de Azevedo LR, Mazur RF, et al: Analysis of the association between lactotransferrin (LTF) gene polymorphism and dental caries. J Appl Oral Sci 18: 166-170, 2010.

9. Bahar B, O'Halloran F, Callanan MJ, McParland S, Giblin L and Sweeney T: Bovine lactoferrin (LTF) gene promoter haplotypes have different basal transcriptional activities. Anim Genet 42: 270-279, 2011.

10. Zhang Y, Lima CF and Rodrigues LR: Anticancer effects of lactoferrin: Underlying mechanisms and future trends in cancer therapy. Nutr Rev 72: 763-773, 2014.

11. Yu MC and Yuan JM: Epidemiology of nasopharyngeal carcinoma. Semin Cancer Biol 12: 421-429, 2002.

12. Stella F, Weich N, Panero J, Fantl DB, Schutz N, Fundia AF and Slavutsky I: Glutathione S-transferase P1 mRNA expression in plasma cell disorders and its correlation with polymorphic variants and clinical outcome. Cancer Epidemiol 37: 671-674, 2013.

13. Velliyagounder K, Kaplan JB, Furgang D, Legarda D, Diamond G, Parkin RE and Fine DH: One of two human lactoferrin variants exhibits increased antibacterial and transcriptional activation activities and is associated with localized juvenile periodontitis. Infect Immun 71: 6141-6147, 2003

14. Moreno-Navarrete JM, Ortega FJ, Bassols J, Castro A, Ricart W and Fernández-Real JM: Association of circulating lactoferrin concentration and 2 nonsynonymous LTF gene polymorphisms with dyslipidemia in men depends on glucose-tolerance status. Clin Chem 54: 301-309, 2008.

15. Cao L, Zhou Y, Li X and Yi H: The relationship of haplotype in lactotransferrin and its expression levels in Chinese Han ovarian cancer. Acta Biochim Biophys Sin (Shanghai) 43: 884-890, 2011.

16. Heo S, Spoerk S, Birner-Gruenberger R and Lubec G: Gel-based mass spectrometric analysis of hippocampal transmembrane proteins using high resolution LTQ Orbitrap Velos Pro. Proteomics 14: 2084-2088, 2014.

17. Haddad T and Kümmerer K: Characterization of photo-transformation products of the antibiotic drug Ciprofloxacin with liquid chromatography-tandem mass spectrometry in combination with accurate mass determination using an LTQ Orbitrap. Chemosphere 115: 40-46, 2014.

18. Zhang JY, Wang F, Zhang H, Lu JQ and Qiao YJ: Rapid identification of polymethoxylated flavonoids in traditional Chinese medicines with a practical strategy of stepwise mass defect filtering coupled to diagnostic product ions analysis based on a hybrid LTQ Orbitrap mass spectrometer. Phytochem Anal 25: 405-414, 2014.

19. Livak KJ and Schmittgen TD: Analysis of relative gene expression data using real-time quantitative PCR and the 2(-Delta Delta C(T)) Method. Methods 25: 402-408, 2001. 
20. Barrett JC, Fry B, Maller J and Daly MJ: Haploview: Analysis and visualization of LD and haplotype maps. Bioinformatics 21: 263-265, 2005

21. Shi YY and He L: SHEsis, a powerful software platform for analyses of linkage disequilibrium, haplotype construction and genetic association at polymorphism loci. Cell Res 15: 97-98, 2005.

22. Niu T: Algorithms for inferring haplotypes. Genet Epidemiol 27: 334-347, 2004

23. Alexander DB, Iigo M, Yamauchi K, Suzui M and Tsuda H: Lactoferrin: An alternative view of its role in human biological fluids. Biochem Cell Biol 90: 279-306, 2012.

24. Nadolska B, Fraczek M, Kręcicki T, Koẹieba M and Zimecki M: Lactoferrin inhibits the growth of nasal polyp fibroblasts. Pharmacol Rep 62: 1139-1147, 2010.

25. Manzoni P, Mostert M and Stronati M: Lactoferrin for prevention of neonatal infections. Curr Opin Infect Dis 24: 177-182, 2011.

26. Gibbons JA, Kanwar RK and Kanwar JR: Lactoferrin and cancer in different cancer models. Front Biosci (Schol Ed) 3: 1080-10888, 2011.

27. Wang J, Li Q, Ou Y, Han Z, Li K, Wang P and Zhou S: Inhibition of tumor growth by recombinant adenovirus containing human lactoferrin through inducing tumor cell apoptosis in mice bearing EMT6 breast cancer. Arch Pharm Res 34: 987-995, 2011

28. Yen CC, Shen CJ, Hsu WH, Chang YH, Lin HT, Chen HL and Chen CM: Lactoferrin: An iron-binding antimicrobial protein against Escherichia coli infection. Biometals 24: 585-594, 2011.

29. Huang C, Wu M, Tang Y, Li X, Ouyang J, Xiao L, Li D and Li G: NAG7 promotes human nasopharyngeal carcinoma invasion through inhibition of estrogen receptor alpha and up-regulation of JNK2/AP-1/MMP1 pathways. J Cell Physiol 221: 394-401, 2009.

30. Raciti M, Lotti LV, Valia S, Pulcinelli FM and Di Renzo L: JNK2 is activated during ER stress and promotes cell survival. Cell Death Dis 3: e429, 2012.
31. Cellurale C, Girnius N, Jiang F, Cavanagh-Kyros J, Lu S, Garlick DS, Mercurio AM and Davis RJ: Role of JNK in mammary gland development and breast cancer. Cancer Res 72: 472-481, 2012.

32. Radovich M, Hancock BA, Kassem N, Mi D, Skaar TC and Schneider BP: Resequencing of the vascular endothelial growth factor promoter reveals haplotype structure and functional diversity. Angiogenesis 13: 211-218, 2010

33. Bruce JP and Liu FF: MicroRNAs in nasopharyngeal carcinoma. Chin J Cancer 33: 539-544, 2014

34. Li HP, Huang HY, Lai YR, Huang JX, Chang KP, Hsueh C and Chang YS: Silencing of miRNA-148a by hypermethylation activates the integrin-mediated signaling pathway in nasopharyngeal carcinoma. Oncotarget 5: 7610-7624, 2014.

35. Ma L, Deng X, Wu M, Zhang G and Huang J: Down-regulation of miRNA-204 by LMP-1 enhances CDC42 activity and facilitates invasion of EBV-associated nasopharyngeal carcinoma cells. FEBS Lett 588: 1562-1570, 2014.

36. Werk AN, Bruckmueller H, Haenisch S and Cascorbi I: Genetic variants may play an important role in mRNA-miRNA interaction: Evidence for haplotype-dependent downregulation of ABCC2 (MRP2) by miRNA-379. Pharmacogenet Genomics 24: 283-291, 2014.

37. Koelsch KA, Webb R, Jeffries M, Dozmorov MG, Frank MB, Guthridge JM, James JA, Wren JD and Sawalha AH: Functional characterization of the MECP2/IRAK1 lupus risk haplotype in human $\mathrm{T}$ cells and a human MECP2 transgenic mouse. J Autoimmun 41: 168-174, 2013.

38. Li Y, Kong D, Ahmad A, Bao B, Dyson G and Sarkar FH Epigenetic deregulation of miR-29a and miR-1256 by isoflavone contributes to the inhibition of prostate cancer cell growth and invasion. Epigenetics 7: 940-949, 2012.

39. Bao L, Hazari S, Mehra S, Kaushal D, Moroz K and Dash S: Increased expression of P-glycoprotein and doxorubicin chemoresistance of metastatic breast cancer is regulated by miR-298 Am J Pathol 180: 2490-2503, 2012. 